\title{
Fibrous Dysplasia of the Thyroid Cartilage - A Case Study and Review of the Literature
}

\section{Aaron Pang*}

The Sainte Chapel Clinic, \#B1-10, Marina Square, 6 Raffles Boulevard, S039594, Singapore

\begin{abstract}
Fibrous dysplasia is a rare disorder of bone that is uncommon in the head and neck region. Herein, we present a rare case of polyostotic fibrous dysplasia involving the right temporal bone and thyroid cartilage in a 19-year-old male. This unique location necessitates close surveillance due to the proximity to the airway and the potential for malignant degeneration.
\end{abstract}

Keywords: Fibrous dysplasia; Thyroid cartilage

\section{Introduction}

Fibrous dysplasia is a rare bone disease that produces $2.5 \%$ of all bony tumors and over 7\% of all non-malignant tumors of bone. We present an uncommon case of polyostotic fibrous dysplasia with involvement of the thyroid cartilage. The clinical features, etiology, histological and radiological features, and treatment of fibrous dysplasia are reviewed.

\section{Case Report}

We report a case of a 19 year old gentleman with no significant past medical or family history of note, who first presented with right-sided headache of 1 month duration. CT scan showed focal bony thickening involving the right squamous temporal bone and greater wing of the sphenoid with internal ground-glass appearance, consistent with focal fibrous dysplasia. He re-presented 5 months later with right-sided cervical lymphadenopathy of 1-month duration. Fine needle aspiration cytology of the cervical lymph nodes showed necrosis with mild acute inflammation. CT scan revealed right-sided suppurative cervical lymph nodes at levels 2 and 3, with an incidental finding of bilateral lamella expansions of the thyroid cartilage with cystic changes on the left, consistent with fibrous dysplasia (Figure 1). Cultures from the aspirate were positive for mycobacterium tuberculosis and the patient was treated with 6 months of anti-tuberculous chemotherapy. He has been followed-up clinically at 6-monthly intervals, and has since remained well, with no further complaints of headache, difficulty in breathing, neck pain or swelling. Follow-up CT scans performed annually have not revealed any growth or malignant transformation of the bony lesions in the temporal and sphenoid bones or thyroid cartilage. To date, no further medical or surgical intervention has been required.

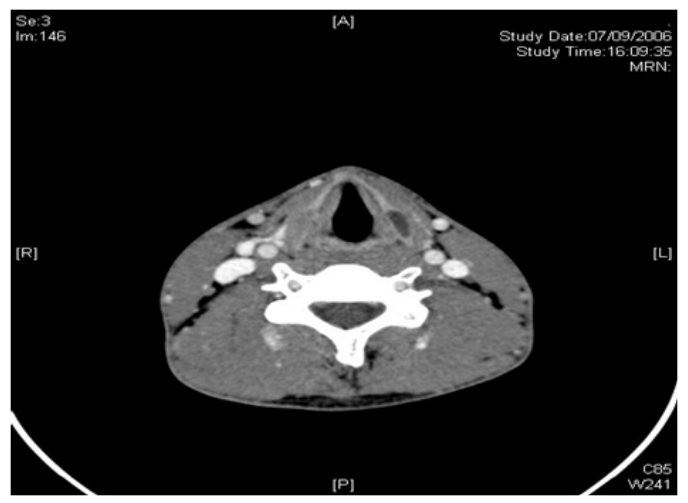

Figure 1: CT scan showing bilateral lamella expansions of the thyroid cartilage with cystic changes on the left.

\section{Discussion}

\section{Clinical features}

Fibrous dysplasia is a non-neoplastic, primary disorder of bone, in which normal medullary bone is replaced with abnormal and structurally weak fibrous and osseous tissue. It is divided into 3 major types: monostotic, involving a single bone; polyostotic, involving multiple bones; and McCune Albright syndrome, a polyostotic form of fibrous dysplasia associated with skin pigmentation and precocious puberty in females.

Fibrous dysplasia presents in children and adolescents, with an average age of onset of 10 years. It manifests as slowly enlarging, painless bony masses. Fibrous dysplasia is relatively rare in the craniofacial region (only $20 \%$ of all locations) and most commonly involves the maxilla and mandible (83.3\%) [1]. To our knowledge, there has been no previous reported case of fibrous dysplasia involving the laryngeal cartilages. The unique location of the tumour in our patient necessitates close follow-up due to the risk of airway compression.

\section{Etiology}

Fibrous dysplasia is caused by a post zygotic somatic mutation of the GNAS1 gene. Acquisition of the mutation early in life leads to a more generalized distribution of the mosaicism and is associated with the classic clinical triad of polyostotic fibrous dysplasia, endocrine hyperfunction, and cafe au lait skin lesions described in McCuneAlbright syndrome. Acquisition of a similar mutation in GNAS1 later in life is associated with the development of isolated lesions (for example, fibrous dysplasia, pituitary or thyroid tumors) [2]. This mutation has been found in the blood, liver and heart in some patients, and cardiac arrhythmias and hepatic disorders have been reported in conjunction with fibrous dysplasia.

Malignant degeneration occurs in $0.5 \%$ of cases of fibrous dysplasia. Malignancies are almost exclusively osteosarcoma.

*Corresponding author: Aaron Pang, The Sainte Chapel Clinic, \#B1-10, Marina Square,6 Raffles Boulevard, S039594, Singapore, Tel: +6563363128; Fax: +6563363968; E-mail: aaron@stchapelleclinic.com

Received November 06, 2013; Accepted January 27, 2014; Published January 29, 2014

Citation: Pang A (2014) Fibrous Dysplasia of the Thyroid Cartilage-A Case Study and Review of the Literature. J Clin Case Rep 4: 335. doi:10.4172/21657920.1000335

Copyright: (c) 2014 Pang A. This is an open-access article distributed under the terms of the Creative Commons Attribution License, which permits unrestricted use, distribution, and reproduction in any medium, provided the original author and source are credited. 
Citation: Pang A (2014) Fibrous Dysplasia of the Thyroid Cartilage-A Case Study and Review of the Literature. J Clin Case Rep 4: 335. doi:10.4172/2165-7920.1000335

Malignant degeneration is greatest in males with polyostotic disease. In monostotic disease, the incidence of malignant degeneration is highest in craniofacial lesions. Radiotherapy increases the risk by 400 times $[3,4]$. The clinical signs of developing malignancy are pain, rapid swelling, and elevation of alkaline phosphatase levels.

\section{Histologic findings}

In fibrous dysplasia, there is replacement of lamellar bone with an abnormal metaplastic version of immature woven bone. There is a matrix of whorls of fibrous tissue surrounding irregular spicules of immature woven bone. The fibrous component is similar to the fibrous stroma of normal immature bone, but the osseous component has irregular borders and lacks the osteoblastic rimming typical of normal bony trabeculae. The irregular, misshapen trabaculae form odd geometric patterns and are described as "chinese letters". In polyostotic fibrous dysplasia, small islands of cartilage are found within the fibrous matrix.

\section{Radiologic findings}

Fries [5] distinguished three types of radiographic appearance for fibrous dysplasia: (1) Pagetoid (56\%); (2) Sclerotic (23\%); and (3) Cystic (21\%).

Pagetoid is the most common and is a mixture of types 2 and 3, with a similar appearance to Paget's disease of bone. These lesions have a "ground glass" appearance with coexisting radiodense and radiolucent areas, due to spicules of new bone. Pagetoid lesions often involve the calvarium. Sclerotic lesions are homogenously dense, while cystic lesions show radiolucency surrounded by a dense rim.

CT reveals a non-homogenous thickening of bone with no soft tissue component. It is helpful in distinguishing fibrous dysplasia from malignancy. Features include osteolysis, destruction of sclerotic lesions, and cortical destruction with soft tissue extension. On T1 MRI, the lesions are non-homogenous with intermediate signal intensity. On T2 MRI, the lesions are non-homogenous with high signal intensity.

\section{Treatment}

Medical treatment includes correction of any underlying endocrine disturbances. Vitamin D and bisphosphonates may help in ameliorating pain and possibly in reconstituting lesions with normal bone. Cortisone has been reported to produce some relief in the pain of bone lesions. Aluminum acetate has been used to precipitate phosphate in the bowel and thus reduce the danger of hyperphosphatemia associated with severe forms of fibrous dysplasia. Hormone therapy has little effect on the course of the disease. Expectant management is based on the possibility that the disease process will quiescent at the onset of puberty.

Indications for surgery are deformity, pathological fractures, pain, compromise of function, and malignancy. Surgical options include conservative bone shaving or radical excision with reconstruction. Surgery may reduce the dangers to vision, hearing, speech or airway caused by the encroachment of tumors.

\section{Conclusion}

Fibrous dysplasia is a rare bone disease which is usually asymptomatic; however its clinical behavior can be severe due to local growth and malignant degeneration. We present the first known case of fibrous dysplasia involving the thyroid cartilage. The unique location of this tumor necessitates close follow-up due to the potential for airway compromise.

\section{References}

1. Ziadi S, Trimeche M, Mokni M, Sriha B, Khochtali H, et al. (2009) [Eighteen cases of craniofacial fibrous dysplasia]. Rev Stomatol Chir Maxillofac 110: 318 322 .

2. Ringel MD, Schwindinger WF, Levine MA (1996) Clinical implications of genetic defects in $\mathrm{G}$ proteins. The molecular basis of McCune-Albright syndrome and Albright hereditary osteodystrophy. Medicine (Baltimore) 75: 171-184.

3. Barat M, Rybak LP, Mann JL (1989) Fibrous dysplasia masquerading as chronic maxillary sinusitis. Ear Nose Throat J 68: 42, 44-46.

4. Edgerton MT, Persing JA, Jane JA (1985) The surgical treatment of fibrous dysplasia. With emphasis on recent contributions from cranio-maxillo-facial surgery. Ann Surg 202: 459-479.

5. Fries JW (1957) The roentgen features of fibrous dysplasia of the skull and facial bones; a critical analysis of thirty-nine pathologically proved cases. Am J Roentgenol Radium Ther Nucl Med 77: 71-88. 